\title{
The circumstellar environment of methanol masers
}

\author{
Chris Phillips \\ JIVE, Postbus 2, 7990 AA Dwingeloo, The Netherlands \\ Douglas Bock \\ RAL, U. California at Berkeley, 601 Campbell Hall, Berkeley, CA 94720 \\ Huib Jan van Langevelde \\ JIVE, Postbus 2, 7990 AA Dwingeloo, The Netherlands \\ Vincent Minier \\ Onsala Space Observatory, 439 92 Onsala, Sweden
}

\begin{abstract}
We present VLBI and BIMA observations of the Class II methanol maser source G 59.783+0.065. The methanol emission arises from the centre of a compact molecular core, supporting the view that methanol masers trace an early stage of massive star formation. The velocity spread of the masers is significantly larger than that of the parent molecular cloud, which is consistent with the methanol coming from a rotating structure within the molecular core. However, there is also weak evidence that the methanol is aligned with an outflow.
\end{abstract}

\section{Introduction}

It is well established that (class II) methanol masers trace regions of massive star formation. There is currently no evidence that methanol masers are associated with other types of objects, though this may be a bias in survey techniques. Observations of MIR and UCHII regions associated with methanol maser sources suggest they trace the earliest stages of massive star formation (Phillips et al 2001; Walsh et al 2001a; Walsh 2001b). However, we know very little about the environment in which methanol masers exist. This limits the amount to which we can use the masers to understand the environment in which the massive star has formed.

One of the more interesting observations of methanol masers is that many sources have a linear morphology, most with a velocity gradient along the source (Norris et al 1998; Phillips et al 1998; Minier, Booth \& Conway 2000). The most exciting explanation is that the methanol emission in these sources delineate an edge on circumstellar disc around a young massive star (Norris et al 1998; Phillips et al 1998). Simple modeling of the velocity gradients gives central masses of between $10-70 \mathrm{M}_{\odot}$. 



Figure 1. EVN observations of the $6.7-\mathrm{GHz}$ methanol masers in G 59.78+0.06. The length scale assumes a (far) kinematic distance of $6.9 \mathrm{kpc}$. Spots represent the (channel averaged) position of individual spectral components found by Gaussian fitting in the image plane and the area is proportional to the peak flux density. The spectrum is derived from these fits and so is weaker than the single dish flux as the individual components are partially resolved.

To improve our understanding of sources which contain methanol masers, high resolution, multi-wavelength observations are needed. High resolution MIR observations hold a lot of promise in this direction (de Buizer 2001) however it is difficult to do accurate astrometry, making accurate comparisons ambiguous.

In this paper we present new VLBI observation of the $6.7-\mathrm{GHz}$ methanol emission towards the source G 59.783+0.065 along with BIMA observations of molecular tracers. This source is within 6" of a source in the IRAS and MSX point source catalogs, but does not have any detectable $8 \mathrm{GHz}$ continuum to a detection limit of $0.3 \mathrm{mJy}$ (Kurtz et al 1994)

\section{Observations}

The $6.7-\mathrm{GHz}$ transition of methanol was observed towards the source $\mathrm{G} 59.78+0.06$ on 14 November 1998 using the European VLBI Network (EVN). The data were processed using AIPS using standard techniques. The restoring beam was $7 \times 6$ mas. 


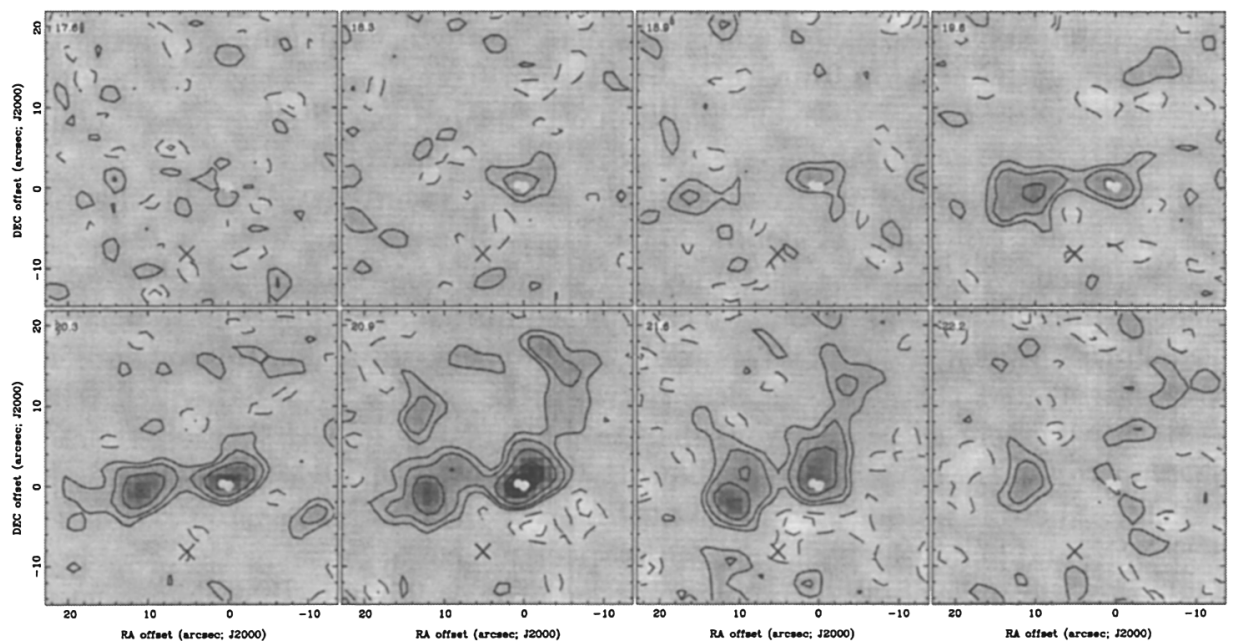

Figure 2. BIMA observations of $\mathrm{HCO}^{+}(1-0)$ towards $\mathrm{G} 59.78+0.06$. The white dots represent the position of the $6.7-\mathrm{GHz}$ methanol maser emission and the black cross is the position of an $\mathrm{H}_{2} \mathrm{O}$ maser.

The millimetre observations were made using the BIMA " $\mathrm{B}$ " array over 2 tracks on 5 \& 6 February 2001 , observing numerous molecular tracers $\left(\mathrm{CH}_{3} \mathrm{CN}\right.$, $\mathrm{SiO}, \mathrm{HC}_{3} \mathrm{~N}, \mathrm{HCO}^{+}, \mathrm{HCN}, \mathrm{CH}_{3} \mathrm{OH},{ }^{13} \mathrm{CS}$ ) and a $100 \mathrm{MHz}$ wide band of continuum. Emission was detected in $\mathrm{HCN}(1-0 \mathrm{~F}=2-1), \mathrm{HCO}^{+}(1-0), \mathrm{HC}_{3} \mathrm{~N}(10-9)$ and $90 \mathrm{GHz}$ continuum. The data were processed using the Miriad package and the restoring beam was $3.7 \times 3.0$ ".

\section{Results and Discussion}

A composite of the VLBI observations of methanol in G 59.78+0.06 is shown in Fig. 1. The size scales are set assuming the source is at the far kinematic of $6.9 \mathrm{kpc}$ (not the near distance of $1.7 \mathrm{kpc}$ ). The far distance has been assumed based on the lack of an UCHII region and the maser spectrum being feature rich but with a relatively weak peak flux density. The eastern and western regions of methanol emission are probably two separate sources, however we cannot rule out the emission coming from two shock fronts on either side of an expanding region. The western cluster has a linear morphology and shows a clear velocity gradient, except for the most northern component which is significantly blue shifted compared to the velocity trend along the source. Assuming Keplerian rotation (taking the velocity gradient along the main source and the disc radius including the northern feature), gives a mass of the central star of $32 \mathrm{M}_{\odot}$. The eastern feature also shows a velocity gradient and an interesting "S" shape.

The BIMA observations of $\mathrm{HCO}^{+}(1-0)$ are shown Fig. 2. The HCN and $\mathrm{HC}_{3} \mathrm{~N}$ images are similar. The methanol emission is aligned with the centre of a compact molecular core. A second molecular core is located 10 arcsec east of the methanol and at a slightly higher central velocity. The methanol is also aligned 
with an unresolved, weak ( $15 \mathrm{mJy})$ continuum source. This flux density is not consistent with emission from an UCHII region (compared to the $8 \mathrm{GHz}$ upper limit) but is consistent with the IRAS $100 \mu \mathrm{m}$ flux density if we are detecting thermal dust emission (and a temperature in the order of $50 \mathrm{~K}$ ).

The two molecular cores could be two sides of an outflow. If this is the case, then it would be strong evidence that the $6.7-\mathrm{GHz}$ methanol maser transition is associated with a outflow regions. However this is thought unlikely. The velocity difference between the two clumps is quite small and both have a narrow $(\sim 4 \mathrm{~km} / \mathrm{s})$ velocity width. We would also expect some continuum emission to be associated the central source between the outflow regions, which is not seen. Most likely these are two separate molecular cores. $\mathrm{HCO}^{+}$often traces outflow, but in the current observations the narrow velocity width of the emission indicates there is no outflow present. However, the observations do not have a high S/N, and high velocity wings on the spectrum could be below the noise level. The other molecules have a similarly narrow profile. We note that these velocity widths are much narrower than the velocity spread across the methanol sources. The gas in which the methanol masers originate must be at a much higher velocity than the ambient velocity within the parent molecular clouds, which is consistent with the methanol emission originating in an embedded rotating structure. The presence of detectable $90 \mathrm{GHz}$ continuum and methanol masers in the western, but not eastern, molecular core indicates the two clouds are at different evolutionary stages.

The western core (with the methanol masers) has a low level extension (seen also in the $\mathrm{HCN}$ and $\mathrm{HC}_{3} \mathrm{~N}$ images) to the north-west while the water maser emission (often associated with outflow) is located diagonally opposite. It is possible that the BIMA observations are detecting one side of an outflow and the water maser the other. The main methanol emission has a similar position angle to this "outflow" which, if real, argues against a disc model for the emission.

\section{References}

de Buizer, J. M. 2001 these proceedings

Kurtz, S. and Churchwell, E. and Wood; D. O. S. 1994, ApJS, 91, 659

Minier, V., Booth, R. S. \& Conway, J. E. 2000, A\&A, 362, 1093

Norris, R. P., Byleveld, S. E., Diamond, P. J., Ellingsen, S. P., Ferris, R. H., Gough, R. G., Kesteven, M. J., et al 1998, ApJ, 508, 275

Phillips, C. J., Smith, C. H., Ellingsen, S. P., Norris, R. P. \& McCulloch, P.M. 2001, MNRAS, in preparation

Phillips, C. J., Norris, R. P., Ellingsen, S. P. \& McCulloch, P. M. 1998, MNRAS, 300,1131

Walsh A. J. 2001a these proceedings

Walsh A. J., Bertoldi, F., Burton, M. G. \& Nikola, T. 2001b MNRAS, in press 